\title{
Il grande complotto televisivo: Giuseppe Genna, Dies Irae (2006)
}

Claudio Milanesi

\section{OpenEdition}

\section{Journals}

Edizione digitale

URL: http://journals.openedition.org/cei/146

DOI: $10.4000 /$ cei. 146

ISSN: 2260-779X

\section{Editore}

UGA Éditions/Université Grenoble Alpes

\section{Edizione cartacea}

Data di pubblicazione: 15 juin 2010

Paginazione: $245-250$

ISBN: 978-2-84310-168-7

ISSN: $1770-9571$

Notizia bibliografica digitale

Claudio Milanesi, «Il grande complotto televisivo: Giuseppe Genna, Dies Irae (2006)», Cahiers d'études italiennes [Online], 11 | 2010, online dal 15 décembre 2011, consultato il 26 mars 2021. URL: http:// journals.openedition.org/cei/146 ; DOI: https://doi.org/10.4000/cei.146 


\title{
IL GRANDE COMPLOTTO TELEVISIVO: GIUSEPPE GENNA, DIES IRAE (2006)
}

\author{
Claudio Milanesi \\ Université d'Aix-Marseille
}

Giuseppe Genna (1969-), scrittore, poeta, critico letterario, pubblicista, la cui produzione può essere seguita sui siti (Clarence, I Miserabili, Carmilla On Line, GiuGenna) con cui collabora o che cura personalmente, ha finora pubblicato 8 romanzi, più una serie di scritti di varia natura (raccolte di saggi, recensioni, instant book). Dei 7 romanzi, 4 formano la serie noir dell'ispettore Lopez. Nei quattro romanzi successivi al ciclo - L'anno luce (2005), Dies Irae (2006), Medium. Una storia vera (2007) e Hitler (2008) ${ }^{\mathrm{I}}-$ Genna esce dal ciclo e dal genere a lui legato. Di questi tre romanzi, Dies Irae è il più impegnativo e ambizioso. Composito, debordante, formato da materiali narrativi incastrati e accatastati, Dies Irae è sovrapposizione di un romanzo di anticipazione, un'autofinzione ${ }^{2}$, un romanzo generazionale sui giovani del post-Sessantotto e un romanzo politico sull'Italia degli anni Ottanta e le sue mitologie, centrato sulla ricostruzione del supposto complotto che starebbe dietro alla morte di Alfredo Rampi, il bambino caduto nel pozzo artesiano di Vermicino il I3 giugno 198I, la cui agonia divenne un memorabile evento televisivo nazionale, la prima diretta della televisione italiana, che seguì per giorni interi i diversi tentativi, tutti falliti, di estrarre il bambino dal pozzo, fino al tragico finale della sua morte. L'idea di fondo del romanzo è che fu la televisione a plasmare la nuova Italia degli anni '8o, l'Italia del riflusso e di Bettino Craxi, l'Italia di Sandro Pertini e del secondo miracolo economico: secondo la ricostruzione che

I. G. Genna, L’anno luce, Milano, Marco Tropea, 2005; Dies Irae, Milano, Rizzoli, 2006; Medium, Milano, Lulu, 2007; Hitler, Milano, Mondadori, 2008.

2. "Récit fondé, comme l'autobiographie, sur le principe des trois identités (l'auteur est aussi le narrateur et le personnage principal), qui se réclame cependant de la fiction dans ses modalités narratives." (Wikipedia, art. Autofiction.) 
ne fa Genna (che lavorò per alcuni anni per conto della Commissione Stragi del Parlamento italiano e che fa riferimento a questa sua esperienza nell'autofinzione del romanzo), il dramma di Vermicino e la sua manipolazione mediatica vennero utilizzati ad arte per occultare, facendoli scivolare fuori dall'attenzione della stampa, la scoperta della loggia massonica P2 (i cui archivi segreti erano stati ritrovati negli uffici di Licio Gelli a Castiglion Fibocchi, vicino Arezzo, dai giudici Colombo e Turone il I7 marzo I98I) e gli scandali ad essa connessi. Gli italiani, dopo la sbornia politica degli anni ' 70 , entravano così nella nuova era in cui, popolazione largamente politicizzata e impegnata direttamente nei conflitti politici e sociali nel periodo che era intercorso fra la nascita della Repubblica e gli anni di piombo, sarebbero diventati progressivamente 50 milioni di spettatori televisivi. I Mondiali di Spagna e la vittoria del 1982 avrebbero poi perfezionato l'opera di questo salto epocale e antropologico. Nella sua ricostruzione di quei giorni del giugno 198I, Genna ricorda che la coincidenza vuole che l'ascesa di Silvio Berlusconi, come costruttore (ma anche come patron di reti televisive) si consolidi nello stesso periodo. Anzi, la pubblicità per la vendita degli immobili di Milano 3 apparve nelle pagine interne del Corriere della Sera proprio il giorno del dramma di Vermicino.

La teoria di Genna è una sorta di complessa visione storico/paranoica che sta fra Marshall McLuhan (il medium è il messaggio) e Pier Paolo Pasolini, per il legame fra salto antropologico e massmedia (negli Scritti corsari, Pasolini identificava nella scuola dell'obbligo e nella televisione gli strumenti della mutazione antropologica). Va ricordato che Genna usa il complotto come principio narrativo (lo ha fatto già in Nel nome di Ishmael, in Non toccare la pelle del drago, e in Grande madre rossa) e l'affabulazione come principio creativo: l'invenzione della realtà è il modo in cui lo scrittore svela quello che rumore mediatico, autocensura, controllo dell'informazione, tendono a occultare ${ }^{3}$. Lo scrittore è sciamano, incanta, spezza e ricombina con la parola le formulazioni della cultura di massa, esacerbandone le mitologie (il Grande Vecchio, la centrale del terrorismo internazionale, il pericolo giallo, ecc.). Questo aiuta a capire perché Dies Irae sia incentrato su un complotto (la "favola verosimile»), dei servizi e dei settori atlantisti che reagisce alla scoperta del complotto precedente (la P2) lanciando una nuova costruzione: la spoliticizzazione dell'opinione attraverso il fatto di cronaca diventato totale grazie all'ubiquità del mezzo televisivo. Qualcuno cala il bambino nel pozzo e nel giro di poche ore le

3. Un esempio di questi giorni è il romanzo The Coming of the Reichchild, Xlibris, dove lo scrittore J. P. de Sales sostiene che Saddam Hussein fosse in realtà il figlio di Adolf Hitler. 
televisioni nazionali lanciano la prima diretta televisiva, che durerà più di ventiquattro ore, coinvolgendo il presidente Pertini, migliaia di curiosi che invadono la conca di Vermicino, gli inviati della stampa scritta e televisiva. La rinascita nazionale (un complotto cha ha come protagonisti, coscienti o involontari, Sandro Pertini, Bettino Craxi, i Mondiali, il riflusso, il tutto organizzato e messo in scena dalla televisione) inizia a Vermicino: gli italiani diventeranno spettatori, la televisione privata finirà nel giro di pochi anni nelle mani di un sol uomo.

Era necessaria questa introduzione per capire quanto il romanzo di Genna sia per la sua essenza stessa un'espressione diretta della tematica delle complesse relazioni fra scrittura e media, fra romanzo e racconto televisivo, fra finzione della parola e realtà dello schermo e inversamente.

Perché questa teoria del complotto attraverso la manipolazione televisiva impone prima di tutto un'evidenza: la televisione appare come un mezzo di tale forza da trasformare l'inconscio collettivo e da diventare elemento plasmante di quello personale. Nel romanzo, il pozzo artesiano di Vermicino diventa parte dell'immaginario del protagonista narrante, e metafora totale (nel senso di Hans Blumenberg), metafora capace cioè di produrre sensi diversi grazie alla sua capacità di essere plasmata e adattata a una moltitudine di contesti: la sofferenza psichica, il trauma infantile, la sterilità comunicativa, l'inabissarsi di un intero modello di società, la vertigine della storia, il rimando continuo di vero e falso, di connivenze e manipolazioni: «L'umanità è serrata nel fango di un pozzo artesiano e fatica a uscirne.» (Dies Irae, p 684.)

Fra i principi adottati per la composizione del romanzo, quello della reiterazione: sequenze narrative, personaggi, eventi, frasi, temi, ritornano a più riprese nel corso della narrazione, in forme e contesti differenti. Frasi pronunciate a Vermicino si ritrovano in bocca a personaggi del romanzo di fantascienza atterrati su un pianeta sconosciuto, metafore come quella del pozzo artesiano segnano l'inconscio di personaggi diversi come il protagonista narrante o Monica, la tossicomane milanese emigrata a Berlino, temi ricorrenti come quello della funzione dei media nell'Italia nell'arco di tempo che va dal 198I a oggi vengono in superficie in diversi momenti del romanzo, come argomento di discussione mondana a una party, come sfogo del protagonista narrante.

Uno dei nuclei problematici è quindi l'interrogativo sul senso del romanzo nella moderna configurazione segnata dall'invadenza dei massmedia nell'immaginario collettivo. Il tema appare una prima volta in occasione dallo scontro fra lo scrittore (Giuseppe Genna rappresenta se 
stesso) e un programmatore televisivo (Massimo) incontrato nel corso di una piccola festa seguita a una rappresentazione teatrale messa in scena dalla troupe di malati mentali ricoverati all'ospedale psichiatrico Paolo Pini. Lo scontro dialettico fra i due personaggi diventa in realtà l'occasione di tematizzare lo stato attuale delle relazioni fra il romanzo e la televisione. Nel romanzo, lo scrittore e il programmatore stanno preparando uno un romanzo l'altro un programma dallo stesso titolo (Dies Irae) basati entrambi su cosiddetti «materiali compromettenti» e finalizzati entrambi a rivelare pezzi occultati della storia politica del Paese. La sfida fra le due forme è così lanciata. E il narratore oscilla fra diverse interpretazioni.

La prima considera che le due forme (il romanzo e il racconto televisivo) si degenerino a vicenda nella ricerca della consolazione del pubblico, nel rispetto delle regole tranquillizzanti dei generi. Da questo punto di vista, romanzo e trasmissione televisiva appaiono ormai come forme decadute di linguaggio. Entrambe sarebbero irreversibilmente diventate forme di comunicazione consolatoria sottoposte agli imperativi del mercato e all'impero dei generi di consumo: gli scrittori «cacano linearità, finzione, televisione stipata nelle pagine di un libro» (Dies Irae, p. 52). Così «scrivere è diventato produrre, lavorare, scalpellare, ornamentare, consegnare una scatola di finte meraviglie» (ibid., p. 90). La degenerazione congiunta delle due forme è particolarmente evidente nel caso del giallo, il genere che è diventato negli ultimi anni imperante tanto nella scena lettararia quanto sugli schermi televisivi, con la moltiplicazione di serie, sceneggiati, film, programmi dove si ricombinano giallo, fatto di cronaca e mistero (o segreto) politico:

[il thriller] è un condizionamento psichico che acceca l'individuo, che canalizza in questo modo, cioè con una suspence da spettatore di tg o da lettore di quotidiano, un desiderio fondamentale $[\ldots]$ quello di conoscere direttamente la verità, non per indagine ma per protagonismo ed esperienza diretta. (Ibid., p. 684.)

La seconda interpretazione tematizza all'opposto la superiorità del romanzo e dello scrittore sulla televisione, per un sovrappiù di «memorabilità e di durata nel tempo» del romanzo rispetto al programma televisivo. In questa formulazione, il romanzo è presentato come una forma di racconto che possiede ancora una forza di durata temporale che la televisione, sottoposta all'effimera volatilità della sua produzione, non ha mai posseduto e possiederà sempre meno. Ma questa superiorità non si potrà consolidare che a condizione che lo scrittore sappia essere pericoloso, che vada «all'attacco sfrontatamente sventolando questa spada di cartapesta che è la letteratura» (ibid., p. 79), che sappia scrivere «un'opera indecrit- 
tabile (che abbia più forme ma che le abbia, queste forme), un'opera che (lettori e committenti) non immaginano, che levi il sospiro e stacchi il filo del respiro, (senza) nessuna concessione al mozzamento di fiato» (ibid., p. 9I). Sempre che uno scrittore così esista:

$\mathrm{Ci}$ vorrebbe una letteratura che spacca ogni genere, ogni gabbia stilistica, ogni poetica con il fantastico. Una letteratura mitologica, delusiva a un primo livello e sapienziale a un secondo, mitologia per questa immane popolazione che fatica a mettersi in movimento perché privata della narrazione di storie mitologiche. (Ibid., p. 685.)

La terza raffigurazione del rapporto romanzo/racconto televisivo è invece implicita nel romanzo stesso, non è quindi tematizzata dal narratore, e contraddice la precedente. Questa terza tematizzazione dello scontro fra le due forme di racconto deriva dai modi e dalla logica stessa del romanzo, e mostra quanto la forza del mezzo televisivo sia ormai profonda. La televisione è ubiqua: nel romanzo, essa appare a ogni snodo del racconto e assume svariate finzioni narrative. È filtro della realtà quando trasmette il dramma di Vermicino: «I canali televisivi Rai trasmettono in diretta euforico ottimismo, $\mathrm{i}$ cronisti inarcano le labbra in un sorriso che comunica più certezza che speranza.» (Ibid., p. I3.) Ne diventa parte integrante, quasi un'applicazione concreta e diretta del principio di indeterminazione: «nel buio illuminato artificialmente dai fari di soccorso e da quelli a uso della televisione, il ronzio pesante dei gruppi elettrogeni che alimentano luce nella notte, la spaccano» (ibid., p. II). Persino la sua assenza produce senso: "Il terremoto in Campania, una tragedia italiana, un'altra tragedia italiana, diversa. Morti, fondi perduti, tangenti, ricostruzioni fantasma, poca tv.» (Ibid., p. I2.) Persino Alfredino, incastrato a 36 metri sotto terra, conosce il mondo attraverso la televisione: quando Elveno Pastorelli, comandante dei vigili del fuoco e futuro segratrio della Protezione Civile, lo chiama da sopra il pozzo, Alfredo lo riconosce: «-Alfredo, sono il comandante dei vigili del fuoco. Il bambino grida - Ti conosco, ti ho visto in televisione» (ibid., p. I2). La televisione è elemento chiave della memoria individuale e collettiva, di una tragedia che unisce il paese e lo unirà nella memoria:

una diretta televisiva infinita, durò ore e ore e ore, a scuola non si parlava altro che di Alfredino e, tornati a casa, subito si accendeva il televisore (se già non era acceso), per partecipare in prima persona agli sviluppi del fatto [...] C'era Pertini [...] Maurizio Vallone [...] Piero Badalon [...] Frajese. (Ibid., p. 35.)

Il racconto televisivo penetra nell'inconscio personale; il Genna scrittore ricorda di aver patito, dopo la diretta di Vermicino, un sogno ricorrente: il piccolo Alfredino che avanza crocifisso in mezzo a una folla immensa: 
Il ragazzino prigioniero nel pozzo, ore e ore e ore a tentare di salvarlo, venne Pertini addirittura, avevo undici anni, ne fui terorizzato. Di notte lo sognai, inchiodato sulla croce, anche nel sogno era notte, il corpicino inchiodato su una croce di legno, la pelle bianchissima e la croce era portata in processione da una folla, una marea di viventi, di cui ricordo ancora certi volti, i particolari. Mi svegliai sudatissimo e la tv era ancora accesa nel salotto, collegata alla scena dei soccorsi a Vermicino, Alfredino era ancora vivo. Ti ricordi? (Ibid., p. 35.)

È un mélange di psichismo e televisione in cui il pozzo artesiano, grazie alla pervasività televisiva diventa forma dell'io. E ancora, adulto, lo scrittore che si autorappresenta ha crisi di claustrofobia quando si trova in un ascensore: «Ogni buca di ascensore mi ricorda un pozzo artesiano. $\mathrm{Mi}$ sento Alfredino, te lo ricordi?» (Ibid., p. 66.) Ma soprattutto, la televisione abilmente manipolata è lo strumento ideale per un'operazione gigantesca di ingegneria sociale, che arriva a trasformare la mentalità e le forme di organizzazione di un intero popolo, trasformato in un aggregato disperso di 50 milioni di spettatori.

Dies Irae è fra l'altro anche il conflitto fra le due forme di racconto, entrambe minacciate dalla banalizzazione consolatoria della plausiblità, ma entrambe in cerca (attraverso i due personaggi Genna e Massimo) della forma di racconto che ancora tracima da questi confini e ancora modifica lettore e spettatore. Nel romanzo, ricco, complesso, contraddittorio, aperto, non vi è soluzione univoca al conflitto, che riaffiora e si articola lungo tutto il romanzo e che il romanzo stesso rappresenta e tematizza. 